\title{
THE EFFECT OF MICROGRAVITY ON THE GROWTH OF SILICA NANOSTRUCTURES
}

D.D. Smith' , L. Sibille ${ }^{2 *}$, R. Cronise', S.J. Oldenburg ${ }^{3 ;}$, D. Wolfe ${ }^{3}$, and N.J. Halas

'Microgravity Sciences and Applications Dept., NASA Marshall Space Flight Center
${ }^{2}$ Universities Space Research Association, NASA Marshall Space Flight Center
${ }^{3}$ ECE Dept. and Dept. of Chemistry, Rice University, Houston, TX 77005

The process of the formation of structures from coagulating ensembles is fundamentally important since the collective behavior of the constituents often results in dramatically improved or unusual mechanical, thermal, chemical, and optical properties. In this study we examine the effect of microgravity on the formation of silica structures, specifically particles and gels.

There is previous evidence that the formation of this "soft" matter is altered in microgravity. The first commercially available products from space (still available from NIST) were the monodisperse latex sphere standards of Vanderhoff $e t a l$. who demonstrated that emulsion polymerization of latexes in space resulted in better monodispersity, increased uniformity and reduced coagulation ( 1 ). In addition it has been hypothesized that in unit gravity, buoyancy driven fluid flows and sedimentation deleteriously perturb sol-gel substructures prior to gelation, and these perturbations are "frozen" into the resulting microstructure (2,3). Wessling et al. (4) have reported that the formation of polyurethane foams in low gravity reduced the average void size, increased the pore roundness, and narrowed the standard deviation in pore size. Leontjev et al. (5) observed fluid flows due to convection and sedimentation during the formation of polyacrylamide gels, and deduced from electrophoretic separations that the resulting pore size distributions were narrower for gels formed in microgravity. More recently Zhu $\mathrm{etal}$. (6.7) have shown that colloidal crystals of polymethylmethacrylate (PMMA) formed in microgravity are an order of magnitude larger and that completely different polymorphs can result. Okubo et al. have studied the kinetics of the formation of colloidal silica particles (both from aqueous silicates and alkoxides) during parabolic aircraft flights using dynamic light scattering and transmission measurements and have found that their formation rate is considerably reduced in microgravity (8).

Stable silica nanoparticle dispersions may be formed either by polymerization of silicic acids or through hydrolysis and condensation of silicon alkoxides (the sol-gel or Stöber route). These two routes are distinguished from one another by the mechanism of particle formation. Comparison of nuclear magnetic resonance (NMR) spectra obtained from Ludox, a commercial aqueous silicate, with acidcatalyzed silicon alkoxides has demonstrated that solutions of the former are dominated by monomers and tetra-functionalized species, whereas di- and tri-functionalized species dominate for alkoxides (9). Moreover, comparison of small-angle x-ray scattering (SAXS) measurements of Ludox with acid-and base-catalyzed alkoxides shows that only aqueous silicate sols are uniform, whereas alkoxides generate fractal particles (10). As Brinker points out (9), these results illustrate that sols derived from aqueous silicates are fully hydrolyzed and grow by classical monomer addition resulting in uniform polymeric

*These authors should be considered of equal importance

Present Address: Seashell Technologies, San Diego, CA 92116

Present Address: Department of Chemistry, Harvard University, Cambridge, MA 02138 

particles, whereas sols derived from silicon alkoxides grow through cluster aggregation and retain a fractal inner morphology even while the particles coarsen through surface tension reorganization.

Two distinct regimes characterize particle growth; diffusion-limited, in which the transport of mass to the growing structure is the dominant limitation to growth; and reaction-limited, in which the efficiency of attachment limits the growth process. These two regimes are universal; the structures formed in one regime are strikingly similar even from vastly different material systems (11). In general, diffusion-limited conditions result in a reduction in the growth rate because there is a decrease in the frequency of collisions. Moreover, those species that do collide do not have the chance to do so in an energetically favorable configuration; i.e. exterior sites are favored. As a result, aggregates formed in diffusion-limited conditions are distinguished by lower fractal dimensions. Reaction-limited growth, on the other hand, is characterized by more compact structures. The sticking coefficient is small enough that species are able to sample attachment sites for energetically favorable configurations.

In this study the formation of silica Stöber particles in microgravity is examined. Microgravity allows diffusion-limited conditions to persist in recipes which typically are reaction-limited, essentially expanding the parameter space under which diffusion-limited conditions prevail, and providing us with a snapshot of the aggregation process that would not normally be accessible. In the case of silica nanostructures, microgravity provides a bias towards diffusion-limited cluster-cluster growth, altering structure formation, and generally resulting in lower fractal dimensions.

Four different recipes were developed in laboratory preparations using the Stöber method $(12,13)$. Silica Stöber particles grown on the ground are of good quality in the range $100-700 \mathrm{~nm}$. However, at certain precursor concentration ratios the particles are either polydisperse, bimodal, rough, or partially aggregated. Hence the recipes were carefully chosen to examine these "failure conditions," essentially spanning a large portion of the parameter space over which Stöber particles may be produced. The first recipe (R1) was a control sample chosen to produce the best possible particles in terms of monodispersity and sphericity. The second recipe (R2) was chosen to produce the smallest Stöber particles, which tend to be rough, irregular, and less monodisperse. The third recipe (R3) was chosen to produce a bimodal size distribution, while the fourth recipe $(\mathrm{R} 4)$ was chosen to produce large irregu$\operatorname{lar}$ (nonspherical) particles. The stoichiometry of each recipe is shown in Table 1 . Note that only recipes $R 3$ and $R 4$ contain additional water.

Table 1. Stiochiometry of silica sol-gel recipes.

\begin{tabular}{ccccc}
\hline Recipe & TEOS $(\mathrm{ml})$ & Ethanol $(\mathrm{ml})$ & Water $(\mathrm{ml})$ & $\mathrm{NH}_{4} \mathrm{OH}(\mathrm{ml})$ \\
\hline R1 & 0.140 & 4.21 & n.a. & 0.654 \\
R2 & 0.153 & 4.59 & n.a. & 0.245 \\
R3 & 0.446 & 3.81 & 0.576 & 0.170 \\
R4 & 0.335 & 3.58 & 0.420 & 0.665 \\
\hline
\end{tabular}

For the space-flight experiment, each $5 \mathrm{ml}$ recipe was divided into two parts and loaded into coupled polyurethane (Hydex) syringes separated by a breakable Parafilm seal to enable mixing of the reactants. The first part consisted of tetraethylorthosilicate (TEOS) and half the ethanol, while the second part consisted of water, ammonium hydroxide $\left(30 \% \mathrm{NH}_{3}\right)$, and the remaining ethanol. Each batch was also divided into ground and space samples, which were stoichiometrically identical. The designated space samples ( 12 total, 3 per recipe) were then placed in the Gelation of Sols; Applied Microgravity Re- 
search (GOSAMR) hardware and activated aboard the space shuttle orbiter (mission STS-95) after microgravity conditions had been established. The GOSAMR hardware, built by 3M Corporation and refurbished for this experiment, essentially consists of a set of modules, each of which contains 8 coupled syringe cartridges. Upon activation a battery-powered motor-driven lead screw with a reversing actuator drives the syringe cartridges back and forth, which mixes the solutions after breaking the barrier seals between them. Upon return of the flight samples, an ultraprobe sonicator was used to obtain diluted suspensions of the samples in ethanol, and these were allowed to evaporate onto carbon coated copper tunneling electron microscope (TEM) grids.

Visual inspection revealed that each of the space-grown samples had formed marginally coherent lowdensity gels, and that these gels coexisted in the syringe with regions of solvent. Ground-grown samples remained in suspension. The resulting ground control particles were only slightly different in size and polydispersity from the laboratory preparations. A dramatic difference between the ground-grown silica structures and those grown in microgravity was observed. Whereas growth in unit gravity produces Stöber particles, growth in microgravity favors loose gel structures. In fact for recipes R 1 and R2 it was difficult to find any Stöber particles at all in the space-grown samples. However, all space-grown samples did form gels, and these gels had a common form and scale which was nearly recipe independent. The particles making up the backbone of the gel were elongated with diameters of approximately $10 \mathrm{~nm}$ and lengths of about $50 \mathrm{~nm}$. These gels are similar to the structures observed by Yoshida (14).

For the recipes containing added water, $\mathrm{R} 3$ and $\mathrm{R} 4$, the particles making up the gel backbone were slightly wider and less elongated. These spheres tended to be smaller and have much larger size distributions than Stöber particles formed on the ground. As shown by Bogush and Zukowski (15,16), the coexistence of large monodisperse spheres $(50-250 \mathrm{~nm})$ with smaller $(\sim 10 \mathrm{~nm})$ aggregating primary particles implies that the growth of silica Stöber particles does not occur by the classical nucleation and growth model, where a fixed number of particles are produced in a single event. Rather Bogush and Zukowski deduced that nucleation of particles proceeds continuously throughout most of the reaction period. The smaller primary particles form by the classical monomer addition growth mechanism and then aggregate because of their small size, until they become colloidally stable. Bogush and Zukoski propose that the resulting stable aggregates are the building blocks for the formation of Stöber particles, collecting smaller aggregates and newly formed particles as they are transported through the solution. Therefore, in this view, reaction-limited conditions must persist to maintain smooth spherical particles. The final structure then coarsens through surface tension reorganization to form the resulting Stöber particle.

In contradiction with Bogush and Zukoski, Harris et al. (17,18) and also van Blaaderen and Vrij (19) have argued that if growth continued to occur through aggregation of subparticles, smooth spherical particles cannot result. In their view Stöber particles initially grow by aggregation of subparticles but monomer addition later fills in the nonuniformities, resulting in a smooth particle. The irregular shape of smaller Stöber particles is a remnant of the aggregation mechanism not yet enveloped by the subsequent monomer growth. The size difference between the subparticles and the resulting Stöber particles certainly supports this view, since this difference is likely too small even for reaction-limited conditions to yield a smooth surface. Furthermore, although only a few Stöber particles formed in microgravity, those that did form were smooth. Hence, the fact that smooth particles are obtained even in the absence of reaction-limited conditions further supports this view.

Thus, it is not valid to consider silica sol-gels as either particulate or polymeric, they are both. The small, $\sim 10 \mathrm{~nm}$, subparticles are primarily polymeric representing the solubility limit of the molecule as a 290 
result of its increasing size and degree of cross-linking [20]. As pointed out by Bailey and Mecartney [21], upon falling out of solution these polymeric precursors collapse, ultimately resulting in a compact particle due to continued hydrolysis and condensation. On the other hand, Stöber particles are at least to some extent particulate, initiated from stable "seeds" formed by aggregation of subparticles, and later smoothed out due to continued addition of monomers.

Colloid stability therefore plays an important role in silica particle formation and morphology to the extent that it determines the size of the aggregates that constitute and augment the particle early in the growth process. According to DLVO theory (22), colloid stability is greatly affected by ionic strength, and the presence of water stabilizes these constitutive aggregates at smaller radii. Hence particle nucleation and growth is more readily established in the presence of water, which in part explains the greater population of Stöber particles in the water-containing recipes $\mathrm{R} 3$ and $\mathrm{R} 4$. In addition, the kinetics of the formation of these aggregates depends on the degree to which the system is diffusionlimited or reaction-limited. Hence microgravity results in a decrease in the rate of formation of these constitutive aggregates due to a bias towards diffusion-limited conditions. Significant monomer depletion (into subparticles and smaller soluble species) then occurs faster than the time it takes for stable aggregates to form and initiate Stöber particle growth, leading to a preponderance of unstable subparticles and aggregates which eventually compose the loosely formed gel that we observe.

Since diffusion is present at any level of gravity, whereas buoyancy driven convection is not, the effect of microgravity on Stöber particle growth can be understood simply through its effect on diffusion. According to the Stokes-Einstein relation, the diffusion coefficient D for particles undergoing Brownian diffusion is

$$
\mathrm{D} \propto \frac{1}{r_{\mathrm{eff}}}=\frac{1}{\mathrm{~m}^{1 / \mathrm{d}(\mathrm{g})}}
$$

where $r_{\text {eff }}$ is the effective radius of the particle, $m$ is the mass, and $d(g)$ is the mass fractal dimension (which depends in some manner on gravitational acceleration $g$ ). Note that the diffusion coefficient only depends on gravity indirectly, through the dependence of the fractal dimension on gravitationally dependent transport mechanisms (convection and sedimentation). Various experiments and computer simulations have demonstrated that in general both diffusion-limited conditions and cluster-cluster aggregation produce more extended structures than reaction-limited monomer growth conditions, resulting in smaller fractal dimensions $(23,24)$. Because in the Stöber route the dominant growth mechanism changes with substructure size, the bias towards diffusion-limited conditions obtained in microgravity leads to a larger decrease in $\mathrm{d}$ for larger substructures. Accordingly, from Equation 1 it can be seen that the diffusion coefficient is reduced to a greater degree for larger substructures (aggregates) in microgravity. Hence the activation barrier for Stöber particle formation is increased in microgravity. For Stöber particles that do manage to form, however, monomer addition again becomes important and the diffusion coefficient of incorporating species increases. The effect of microgravity is therefore to further increase the difference in the growth rates for different growth mechanisms. Equivalently, microgravity suppresses the coagulation of subparticles and aggregates more dramatically than it does their formation through addition of monomers and other small soluble species.

The importance of the aggregation of unstable clusters and subparticles to the formation and growth of silica Stöber particles makes the effect of microgravity on Stöber particle growth profound. Rather than simply retarding structure growth (in this case a silica sol) as would be expected for a singular growth mechanism, a pathway to an entirely different structure becomes available. Microgravity favors diffusion-limited conditions, which slows the formation of stable particle-forming aggregates. Monomers are 
consumed more by unstable subparticles and aggregates than by Stöber particles. Eventually clustercluster aggregation is the only remaining growth mechanism which yields more extended structures leading ultimately to gel formation. These results suggest that microgravity favors the formation of more rarefied sol-gel structures, providing a bias towards diffusion-limited cluster-cluster aggregation.

\section{REFERENCES}

1. J.W. Vanderhoff, M.S. El-Aasser, F.J. Micale, E.D.Sudol, C.M. Tseng, and H.R. Sheu, ACS Polymer Preprints 28, 455 (1987); Mater. Res. Soc. Symp. Proc. 87, 213 (1987).

2. D.A. Noever, Microgravity Science and Technology 3, 14 (1994).

3. L. Sibille, R.J. Cronise, D.A. Noever, and A.J. Hunt, Proc. Space Technology and Applications International Forum, $I^{\text {st }}$ Conference on Commercial Development of Space, Albuquerque, January 7-11, p. 451, 1996.

4. F.C. Wessling, S.P. McManus, J. Mathews, and D. Patel, J. of Spacecraft and Rockets 27 (8), 324 (1990).

5. V.B. Leontjev, Sh.D. Abdurakhmanov, M.G. Levkovich, "Study of polyacrylamide gels synthesized during microgravitation," Proc. AIAA Microgravity Science Symposium, Moscow, May 13 - 17. 274 (1991).

6. J.X. Zhu, M. Li, S.E. Phan, W.B. Russel, P.M. Chaikin, R. Rogers, M. Meyer, "Dynamics of disorder-order transitions in hard sphere colloidal dispersions in microgravity," $3^{\text {rl }}$ Microgravity" Fluid Physics Conference, CP 3338, 397 (1996).

7. J.X. Zhu, M. Li, R. Rogers, W. Meyer, R.H. Ottewill, STS-73 Space Shuttle Crew, W.B. Russel, P.M. Chaikin, Nature 387, 883 (1997).

8. T. Okubo, A. Tsuchida, K. Kobayashi, A. Kuno, T. Morita, M. Fujishima, and Y. Kohno, Colloid. Poym. Sci. 277, 474 (1999).

9. C.J. Brinker, in The Colloid Chemistry of Silica, H.E. Bergna, Ed. (American Chemical Society, Washington, 1994), p. 361.

10. D.W. Schaefer, J.E. Martin, and K.D. Keefer, in Physics of Finely Divided Matter, N. Bocarra and M. Daoud, Eds. (Springer-Verlag, Berlin, 1985), p. 31.

11. M.Y. Lin, H.M. Linday, D.A. Witz, R.C. Ball, R. Klein, P. Meakin, Nature 339, 360 (1989).

12. W. Stöber, A. Fink, and E. Bohn, J. Colloid Interface Sci. 26, 62 (1968)

13. R. D. Averitt, D. Sarkar, and N.J. Halas, Phys. Rev. Lett. 78, 4217 (1997).

14. A. Yoshida in The Colloid Chemistry of Silica, H.E. Bergna, Ed. (American Chemical Society, Washington, 1994), p. 51.

15. G.H. Bogush and C.F. Zukoski, Proc. of the $44^{\text {th }}$ Annual Meeting of the Electron Microscopy Society of America, G.W. Bailey, ed. (San Francisco Press, San Francisco, 1986), p. 846.

16. G.H. Bogush and C.F. Zukoski in Ultrastructure Processing of Advanced Ceramics, J.D. Mackenzie and D.R. Ulrich, eds. (Wiley, New York, 1988), p. 477.

17. M.T. Harris, O.A. Basaran, and C.H. Byers, in Ultrastructure Processing of Advanced Ceramics, J.D. Mackenzie and D.R. Ulrich, Eds. (Wiley, New York, 1998), p. 843.

18. M.T. Harris, R.R. Brunson, and C.H. Byers, J. Non-Cryst. Solids 121, 397, (1990).

19. A. van Blaaderen and A. Vrij in The Colloid Chemistry of Silica, H.E. Bergna, Ed. (American Chemical Society, Washington, 1994), p. 83.

20. P.J. Flory, Principles of Polymer Chemistry (Cornell University Press, Ithaca NY, 1953).

21. J.K. Bailey and M.L. Mecartney, Colloids and Surfaces 63, 151 (1992).

22. E.J.W. Verwey and J.Th.G. Overbeek, Theory of the Stability of Lyophobic Colloids (Elsevier, New York, 1948).

23. P. Meakin in On Growth and Form, H.E. Stanley and N. Ostrowsky, Eds. (Martinus-Nijhoff, Boston, 1986), p. 111.

24. P. Meakin, Ann. Rev. Phys. Chem. 39, 237 (1988). 